\title{
Effects of Electromagnetic Fields on the Hippocampus
}

\author{
Asghar Rajabzadeh ${ }^{1, *}$ \\ ${ }^{1}$ Department of Anatomical Sciences, Faculty of Medicine, Lorestan University of Medical Sciences, Khorramabad, Iran \\ "Corresponding author: Department of Anatomical Sciences, Faculty of Medicine, Lorestan University of Medical Sciences, Khorramabad, Iran. Email: \\ dr.a_rajabzadeh@yahoo.com
}

Received 2018 February 26; Revised 2018 September 08; Accepted 2018 September 26.

Keywords: Electromagnetic Fields, Hippocampus, Oxidative Stress

\section{Dear Editor,}

Electromagnetic fields (EMFs), physical energy, are non-ionic waves with speed, frequency, wavelength, and amplitude characteristics, emitted by electrical machines and industry equipments. According to frequency, these fields are divided to low, intermediate, high, and static radiations. In previous studies, it has been claimed that EMFs (duration of exposure) have noxious outcomes on biological systems of humans and rodent, based on frequency and other properties $(1,2)$. Most researches have been done on EMFs and central nervous systems (CNS) with different exposures involved in vital portions $(3,4)$.

Hippocampus formations, as the main compartment of the limbic system, are principal members of CNS at emotional, behaviors, memory, and regulating of endocrine systems, which could be one of the high sensitive portions to EMFs waves. According to previous studies, it is estimated that EMFs have remarkable effects on neurological compartments (5). A previous study by the current authors showed that $30 \mathrm{mT}$ electromagnetic fields has negative impacts on rat's hippocampus (6). Increased free radicals and created oxidative stress via EMFs waves may be one of the most important reasons (7). This condition leads to promotion of cell's apoptosis pathway in the main parts of hippocampus, such as the dentate gyrus (6).

The EMFs have a potential role in the starting of intrinsic and extrinsic pathways of apoptotic process. Breaking of DNA in brain's cells, chromosome abnormalities, genetic mutations, intracellular enzymes dysfunction, and altered expression level of neurotransmitters in various parts of the brain are side effects of electromagnetic fields $(8,9)$. Furthermore, it is assumed that EMFs have a major effect on high metabolic and proliferative organs, such as hippocampus formations. The produced free radicals in the hippocampus and similar structures can be defined as sensitive organs to EMFs (Figure 1).
However, in addition to some researches on the relationship between electromagnetic fields and CNS, there is no suitable strategy to remove electrical devices in the society's life style. In this case, general global designs should be made in order to prevent abnormal births and the risk of neurological disorders.

High production of free radicals as a result of EMFs waves may have important effects on the hippocampus, which leads to genetic mutation, DNA breakage, and apoptosis in the cell's lineage.

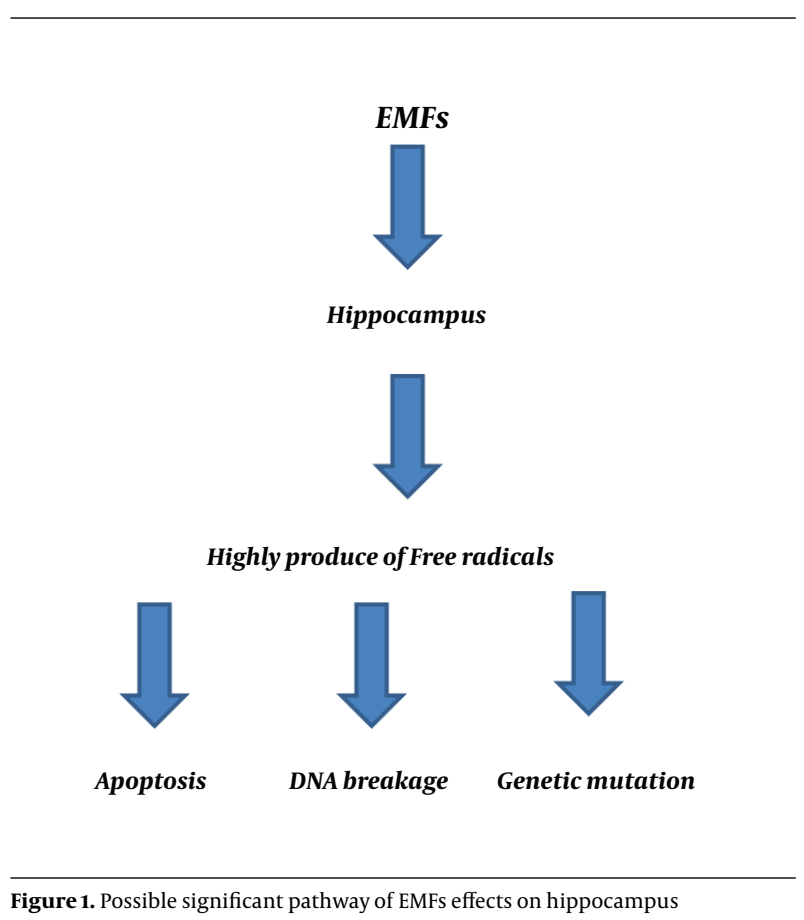

Figure 1. Possible significant pathway of EMFs effects on hippocampus 


\section{References}

1. Asghari A, Khaki AA, Rajabzadeh A, Khaki A. A review on electromagnetic fields (EMFs) and the reproductive system. Electron Physician. 2016;8(7):2655-62. doi: 10.19082/2655. [PubMed: 27648194]. [PubMed Central: PMC5014506].

2. Miyakoshi J. Biological responses to extremely low frequency electromagnetic fields. J Dermatol Sci. 2006;2:23-30. doi: 10.1016/j.descs.2006.08.003.

3. Soleimani R, Allahvaisie O, Khaki AA. [Electromagnetic field effects on rat's cerebella ultrastructure]. Sci J Kurdistan Univ Med Sci. 2006;11:1-9. Persian.

4. Lai H, Singh NP. Magnetic-field-induced DNA strand breaks in brain cells of the rat. Environ Health Perspect. 2004;112(6):687-94. doi: 10.1289/ehp.6355. [PubMed: 15121512]. [PubMed Central: PMC1241963].

5. Friedman J. The role of free radicals in the nervous system. In: Gadoth $\mathrm{N}$, Hilmar Gobel $\mathrm{H}$, editors. Oxidative stress and free radical damage in neurology. Springer; 2011. p. 1-17.

6. Teimori F, Khaki AA, Rajabzadeh A, Roshangar L. The effects of 30 mT electromagnetic fields on hippocampus cells of rats. Surg Neurol Int. 2016;7:70. doi: 10.4103/2152-7806.185006. [PubMed: 27453795]. [PubMed Central: PMC4946261].

7. Simonian NA, Coyle JT. Oxidative stress in neurodegenerative diseases. Annu Rev Pharmacol Toxicol. 1996;36:83-106. doi: 10.1146/annurev.pa.36.040196.000503. [PubMed: 8725383].

8. Robison JG, Pendleton AR, Monson KO, Murray BK, O’Neill KL. Decreased DNA repair rates and protection from heat induced apoptosis mediated by electromagnetic field exposure. Bioelectromagnetics. 2002;23(2):106-12. doi:10.1002/bem.103. [PubMed: 11835257].

9. Mausset AL, de Seze R, Montpeyroux F, Privat A. Effects of radiofrequency exposure on the GABAergic system in the rat cerebellum: Clues from semi-quantitative immunohistochemistry. Brain Res. 2001;912(1):33-46. doi:10.1016/s0006-8993(01)02599-9. 